\title{
A Case Report and a Descriptive Retrospective Study of Cases of Inferior Vena Cava Thrombosis Diagnosed over a 10-Year Period in a Third Level Hospital
}

\author{
Andrea Teira1, Mireia Menéndez ${ }^{2}$, Zaida Salmón ${ }^{3}$, Daniel Nan ${ }^{3}$ \\ ${ }^{1}$ Department of Cardiology, Hospital Universitario Marqués de Valdecilla, Santander, Spain \\ ${ }^{2}$ Department of Oncology, Hospital Universitario Marqués de Valdecilla, Santander, Spain \\ ${ }^{3}$ Department of Internal Medicine, Hospital Universitario Marqués de Valdecilla, Santander, Spain \\ Email: andrea.teira@scsalud.es
}

How to cite this paper: Teira, A., Menéndez, M., Salmón, Z. and Nan, D. (2020) A Case Report and a Descriptive Retrospective Study of Cases of Inferior Vena Cava Thrombosis Diagnosed over a 10-Year Period in a Third Level Hospital. Case Reports in Clinical Medicine, 9, 122-130.

https://doi.org/10.4236/crcm.2020.95018

Received: March 24, 2020

Accepted: April 21, 2020

Published: April 24, 2020

Copyright $\odot 2020$ by author(s) and Scientific Research Publishing Inc. This work is licensed under the Creative Commons Attribution International License (CC BY 4.0).

http://creativecommons.org/licenses/by/4.0/ (c) (i) Open Access

\begin{abstract}
Inferior vena cava thrombosis is an under-recognized entity associated with significant morbidity and mortality. This is the reason why, although the diagnosis is challenging, a high index of suspicion is required. Regarding this condition, we present the case of a 63-year-old man who had repeatedly visited the emergency room suffering from abdominal and back pain and painful lower limb edema. After several tests, including magnetic resonance imaging (MRI), he was diagnosed to have agenesis of left renal vein and inferior vena cava thrombosis, from hypercoagulable state secondary to Antiphospholipid Syndrome. He had anticoagulation treatment with low-molecular-weight heparin with good subsequent evolution. This study sets out a descriptive retrospective study of fifty cases of inferior vena cava thrombosis diagnosed in a third-level hospital in the north of Spain over a ten-year period (2010-2018). The aim of this article is to identify the epidemiology, predisposing factors and symptoms that characterize this entity, in order to be able to achieve an early diagnosis that allows us to initiate immediate treatment, minimizing acute and chronic complications of this disease.
\end{abstract}

\section{Keywords}

Inferior Vena Cava Thrombosis, Antiphospholipid Syndrome, Morphologic Vascular Variant, Hypercoagulable State, Anticoagulation, Imaging Techniques, Descriptive Retrospective Study 


\section{Introduction}

Inferior vena cava (IVC) thrombosis is a rare disease that may be underestimated due to the lack of standardized methods for its detection and to the fact that it can be clinically silent. Lifetime incidence for deep vein thrombosis is $0.1 \%$ and around $4 \%-15 \%$ of these cases will develop IVC [1] [2]. The importance of these data lies in these two facts; the high morbidity associated to this condition, with complications such as post thrombotic syndrome or pulmonary embolism [3] [4] [5], and the double mortality rate of IVC thrombosis compared to isolated lower limb deep vein thrombosis.

This great impact on patient's life outlines the importance of this condition. In this review we outline etiology, symptoms, treatment, prognosis and other factors such as epidemiology of IVC thrombosis in fifty cases diagnosed in a third-level hospital in the north of Spain from 2010 to 2018.

\section{Case Report}

A 63-year-old man who had repeatedly visited the emergency room for 2 months suffers from abdominal and back pain as well as lower limb paresthesias and painful leg swelling. Regarding his medical records, he was a smoker diagnosed of hypertension and stage 3 chronic kidney disease.

He was admitted to the hospital due to the persistence of the symptoms. Laboratory tests revealed creatinine $1.69 \mathrm{mg} / \mathrm{dL}$, PCR $9.2 \mathrm{mg} / \mathrm{dL}$ and VSG $55 \mathrm{~mm}$. Clinical examination confirmed painful bilateral lower limb edema.

Lower limb Doppler ultrasound was performed and deep vein dilatation was demonstrated without evidence of thrombosis. Bearing in mind that the patient was allergic to iodine contrast and had chronic kidney disease, a basal computerized tomography $(\mathrm{CT})$ was performed revealing agenesis of left renal vein and indirect signs of thrombosis such as swelling of abdominal adipose tissue [Figure 1].

We completed imaging study with magnetic resonance imaging (MRI) in which agenesis of left renal vein and extensive inferior vena cava thrombosis up

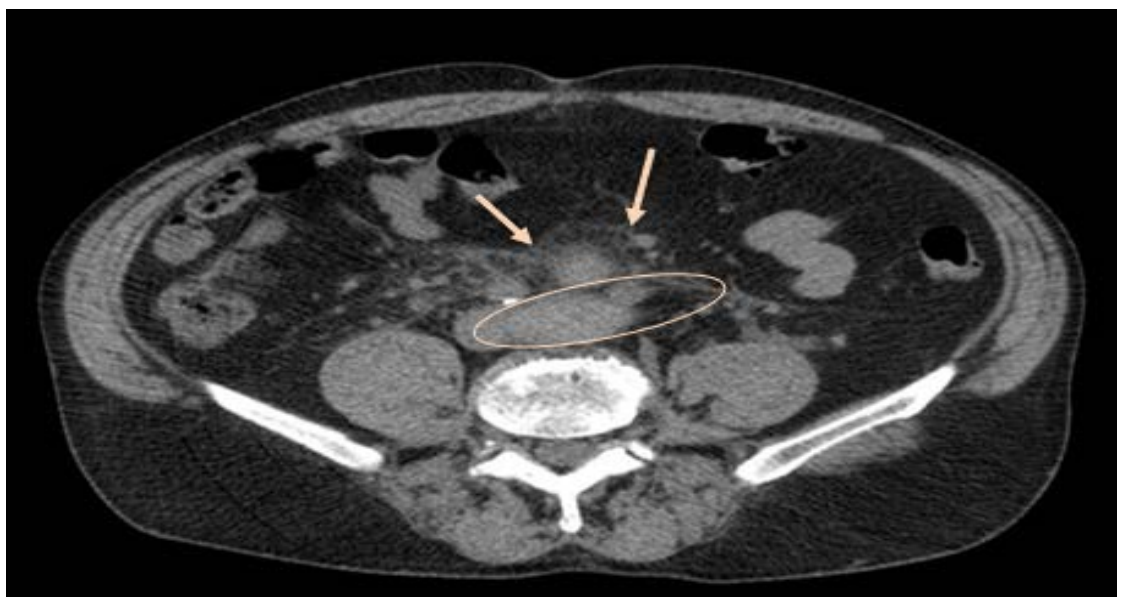

Figure 1. Oval image well defined adjacent to retroperitoneal vessels of $4 \mathrm{~cm}$ wide surrounded by swelling of abdominal adipose tissue. 
to right renal vein and bilateral iliac vein thrombosis were confirmed [Figure 2].

Further tests were performed, and the patient was diagnosed of a hypercoagulable state secondary to antiphospholipid syndrome.

Conservative treatment with low-molecular-weight heparin was initiated and after 10 days of hospitalization and appropriate progress, he was discharged. During follow up, the patient remains stable and continues anticoagulation treatment with vitamin $\mathrm{K}$ antagonist.

\section{Methods}

This article displays a descriptive retrospective study of cases of inferior vena cava thrombosis, diagnosticated over a period of 10 years in a third level hospital, from January 2010 to August 2018.

The patient's data were analyzed based on epidemiologic features, location of thrombosis, predisposing factors, clinical features, diagnostic techniques, treatment and complications due to thrombosis and those related to therapeutic anticoagulation. The analytic tool used was SPSS v.14.0.

The patients younger than 18 years old and those cases in which there was no evidence of inferior vena cava thrombosis in their medical record were excluded from the analysis.

\section{Results}

From 90 potentially relevant cases identified at the beginning, 40 of them met exclusion criteria so were ruled out. Finally, 50 cases were included for the analysis. Main results are summarized in Table 1.

The sample used for this work included 50 patients with an average age of 65.3 years ranging from 20 to 88 years. There was equal gender distribution, with female-male sex ratio 1:1 [Table 1].
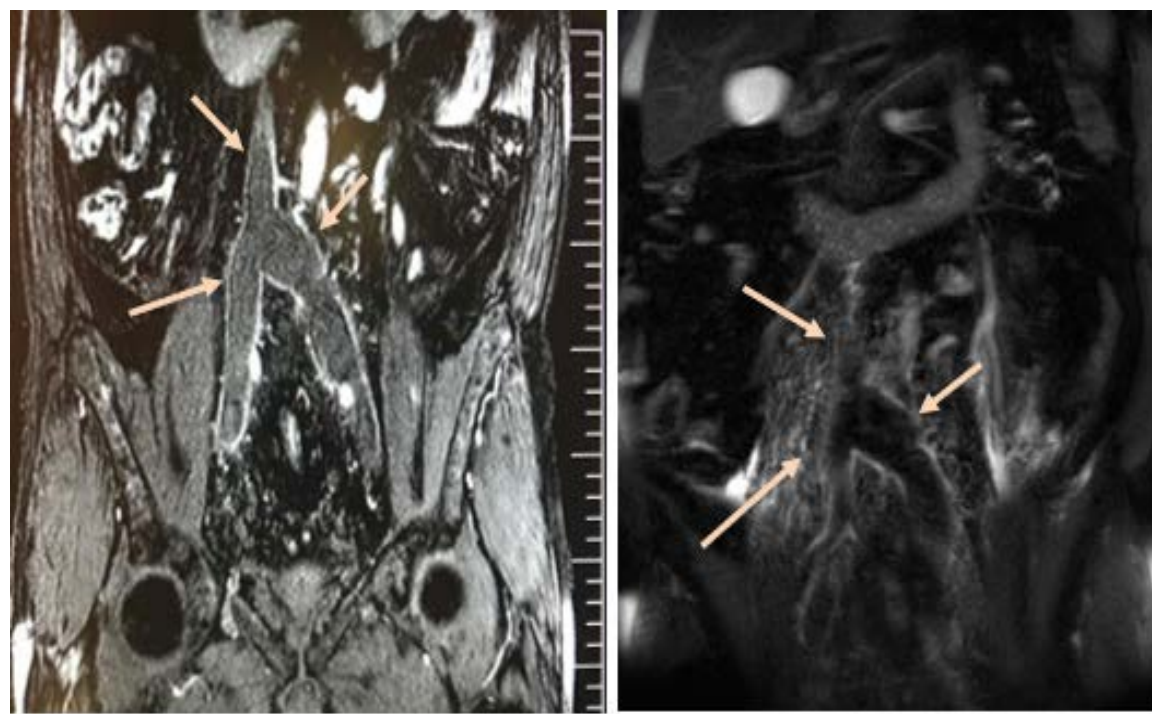

Figure 2. Coronal images of inferior cava \& iliac vein thrombosis; T1 sequency MRI (left), T2 sequency MRI (right). 
Table 1. Epidemiologic characteristics; risk factors; onset symptoms; imaging techniques; and complications during follow up.

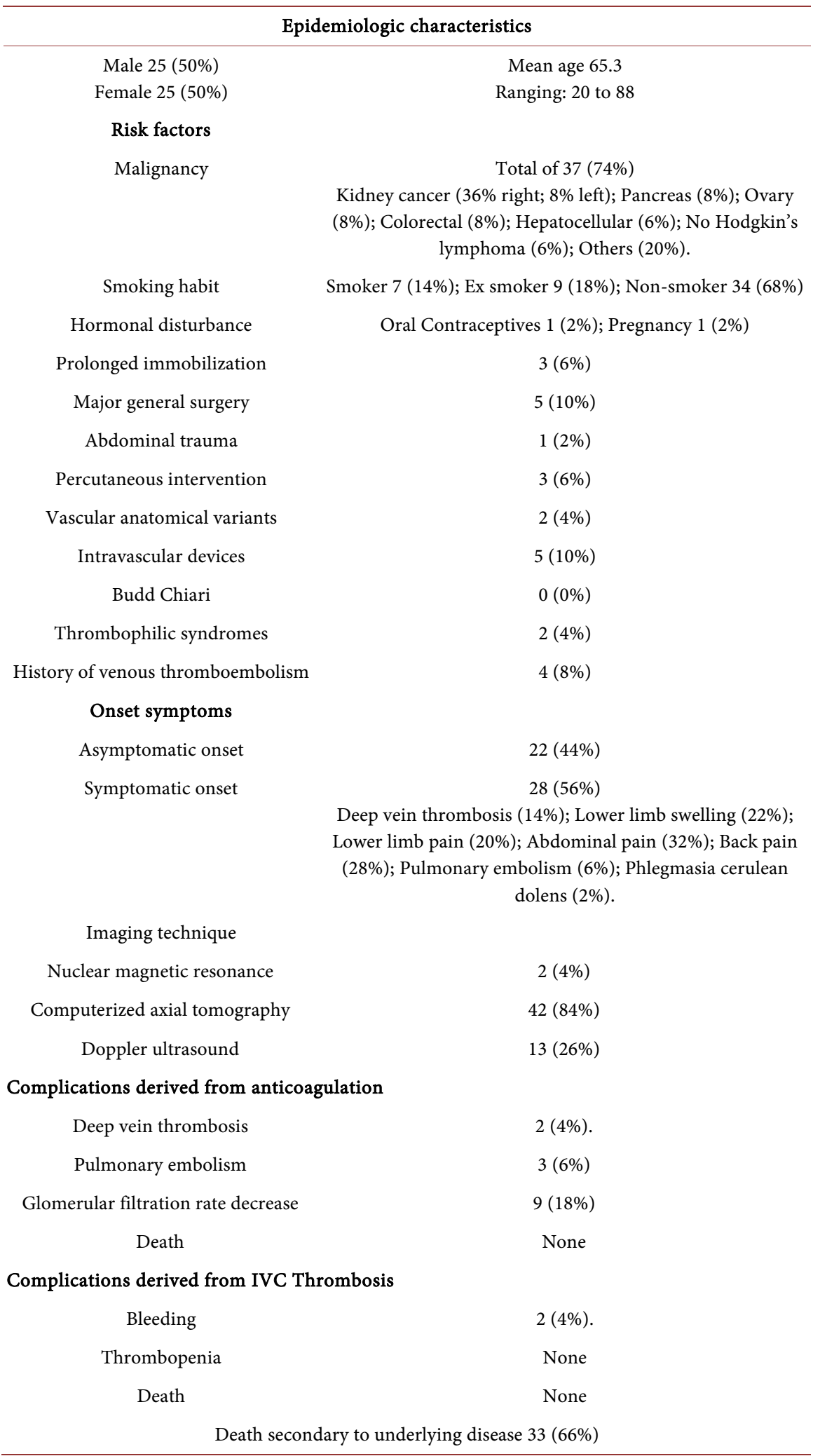

DVT $=$ Deep Vein Thrombosis, IVC = Inferior Vena Cava, Immobilization, Mayor General Surgery, Abdominal trauma or Percutaneous intervention $<1$ month. 
Isolated IVC thrombosis was found in $20 \%$ of all cases. The most frequent location was inferior vena cava including iliac vein thrombosis (38\%); followed by inferior vena cava including renal vein thrombosis $(26 \%)$. Left renal vein involvement was more frequent (16\%), than right renal vein thrombosis $(8 \%)$. There was one case of bilateral renal vein thrombosis $(2 \%)$. In one case pulmonary embolism was the first clinical manifestation (2\%).

Risk factors, symptoms and diagnostic imaging techniques are summarized in Table 1.

The strongest predisposing factor for IVC thrombosis was malignancy. In fact, $74 \%$ also had concomitant malignancy at the time of the thrombosis. In $68 \%$ of the cases the diagnosis of both (IVC thrombosis and cancer) was synchronic, so only $6 \%$ had been treated with antitumoral therapy. Among all malignancies, renal cell carcinoma was the most prevalent tumor (36\% were right renal carcinoma; and $8 \%$ were left renal carcinoma).

A total of 22 cases were asymptomatic at the time of the diagnosis (44\%). On the rest, the most common onset symptoms were lower limb pain and swelling, abdominal pain and low back pain [Table 1].

Regarding treatment, 41 patients $(82 \%)$ initiated anticoagulation with lowmolecular-weight heparin. A total of 24 patients continued this treatment (48\%) and 17 (34\%) switched to anti-vitamin K drugs. No patient received a directacting anticoagulant agent. Treatment duration was less than 6 months in 17 patients (34\%) and 24 patients (48\%) received anticoagulation for over 6 months.

Side effects in relation to either thrombosis or anticoagulation therapy are both summarize in Table 1 .

\section{Discussion}

\section{Risk factors}

The etiology of Inferior vena cava thrombosis can be either congenital or acquired.

In one hand, one of the causes of congenital IVC thrombosis is vascular anatomical variants. In general population the prevalence is $0.5 \%-1 \%$, especially in men in their $30 \mathrm{~s}$ and $40 \mathrm{~s}$ [3] [6]. Regarding the case of study, our patient was diagnosed of agenesis of left renal vein at the time of the thrombosis. In our series, two of the cases reviewed suffered from vascular anatomical variants (double right renal vein and independent suprarenal cava vein with an anomalous drainage). These data correspond to the $4 \%$ of all cases in our analysis, which doubles the prevalence reported in the general population.

On the other hand, acquired IVC thrombosis can be caused by spontaneous thrombosis, by external compression, by malignancy or due to pathological changes in the vessel's wall [3].

In relation to malignancy, as reported in medical literature, there is a procoagulant state induced by the tumor itself and by most of antitumoral treatments. In fact, in our analysis, suffering from an active tumor is the strongest predis- 
posing factor for developing inferior vena cava thrombosis, kidney cancer being the most prevalent tumor. In addition, we reported that right side renal carcinoma is more prevalent than left sided ( $36 \%$ and $8 \%$ respectively).

Another procoagulable state, and therefore risk factor for IVC thrombosis, are thrombophilias. Antiphospholipid Syndrome was the hypercoagulable state that induced IVC in our case of study. Regarding our analysis, two patients ( $4 \%$ of all cases) had an associated thrombophilic syndrome; in one case it was antiphospholipid syndrome and in the other one it was prothrombin 20210A gene mutation.

Smoking is also a well-known prothrombotic factor [3] [5]. In our analysis, $32 \%$ of all patients were smokers or ex-smokers.

Interventional procedures, abdominal surgery, abdominal trauma and prolonged immobilization belong to the list of acquired risk factors for thrombosis associated with inferior vena cava thrombosis. At least one of the previously listed was reported in the $20 \%$ of the patients in our analysis.

Placement of intravascular foreign bodies is also a predisposing factor for thrombosis [3]. About $10 \%$ of all the patients from our review carried an intravascular device (three IVC filters, one ECMO and one femoral vein catheterization).

The prevalence of previous deep vein thrombosis treated with anticoagulation therapy in our analysis was up to $8 \%$.

\section{Symptoms}

In relation to the clinical onset, as it is reported in medical literature, it can range from asymptomatic to hemodynamic instability [3] [7] [8]. In our analysis, $44 \%$ of all patients were asymptomatic and diagnosis was incidentally. In symptomatic cases, most frequent clinical manifestations were leg swelling (22\%), lower limb pain (20\%), low back pain (20\%) and abdominal pain (32\%). In 6\% of all cases pulmonary embolism was the first clinical manifestation.

One case of phlegmasia cerulean dolens was reported. This severe condition is associated with cyanosis and high risk of venous gangrene and limb amputation [9] [10].

\section{Diagnosis}

Due to the lack of and non-specific clinical manifestations and insidious onset, diagnosis of IVC thrombosis is based on imaging techniques. Doppler ultrasound is recommended in patients with lower extremity symptoms. However, thrombus in infrarenal cava can be difficult to identify. The best diagnostic choice to accurately assess the presence, size and location of IVC thrombosis is CT or MRI, both using intravenous contrast [11].

Considering both techniques; on one hand, CT is quicker, non-invasive and can accurately diagnose and assess extension of thrombosis as well as any associated local abnormalities. On the other hand, MRI avoids radiation and provides more accurate definition of thrombus. However, cost, accessibility and risk of nephrogenic systemic fibrosis when gadolinium use, makes CT the most pop- 
ular non-invasive imaging tool in the medical literature and therefore the technique of choice for diagnosis.

In our analysis $84 \%$ of all 50 cases were diagnosed by CT, Doppler ultrasound was performed in $14 \%$ and only $4 \%$ of cases where diagnosed by MRI. CT was the technique of choice probably due to its accessibility.

\section{Treatment}

Goals of treatment are minimizing mortality, sequelae such as pulmonary embolism, risk of recurrence and thrombus persistence, preventing chronic episodes [10].

Few data are reported in medical literature, but anticoagulation therapy seems to be the best choice even though the optimal therapeutic strategy remains unclear. Other therapies, such as endovascular treatment or catheter-directed treatment, should be considered in the case of acute presentation $(<14$ days $)$ or high risk of bleeding [3] [5] [7] [10].

Just as it is reported in the medical literature, our analysis showed that a large proportion $(80 \%)$ of the patients we reviewed initiated anticoagulant therapy with a low molecular weight heparin. More patients remained on treatment with these agents that were switched to an antivitamin $\mathrm{K}$ drug, due to the large number of cases with active tumors. In accordance with the lack of published experience with direct-acting anticoagulant agents, none of the patients in our series was treated with one of these drugs.

Length of treatment seems to have been driven by associated conditions and malignancy-related survival. No conclusion or recommendation can be extracted from our experience or from what has been published.

Due to the high risk of bleeding, surgical thrombectomy was performed in $6 \%$ of all cases with favorable outcome.

Only $12 \%$ among all patients did not receive any treatment: three (6\%) because thrombosis was partial, one (2\%) due to extremely high risk of bleeding, and two (4\%) because palliative management was decided.

In relation to anticoagulation therapy complications, in our analysis, $4 \%$ of all cases developed bleeding in relation to anticoagulation therapy. None of them was fatal and both patients had previous history of bleeding. No thrombocytopenia was reported. Summing up, it seems that anticoagulation is a safe treatment in inferior vena cava thrombosis.

Finally, in our analysis the main cause of death was secondary to the patient's underlying disease (66\%), mainly oncological. None among all the patients died directly as a consequence of IVC thrombosis.

\section{Conclusions}

Malignancy seems to be the predisposing condition most strongly associated with inferior vena cava thrombosis, although it is also related with anatomic vascular variants and prothrombotic risk factors.

Signs and symptoms range from asymptomatic to cardiovascular collapse. Pain- 
ful lower limb swelling, abdominal pain and lower back pain are suggestive of IVC thrombosis.

Due to the lack of and non-specific clinical manifestations and insidious onset, diagnosis of IVC thrombosis is based on imaging techniques. Computerized axial tomography or magnetic resonance is useful for diagnosis, but computerized axial tomography is preferred probably because of its accessibility. Doppler ultrasound should be used as screening test in case of lower limb symptoms (swelling, pain).

Treatment is adapted to each case and can range from anticoagulation and lower limb compression to invasive treatments. In our analysis, anticoagulation with low-molecular-weight heparin was the most used treatment, bearing in mind that most of the cases were in relation to malignancy. No patient was treated with a direct-acting anticoagulant agent. Treatment duration was varied and very influenced by the underlying diseases and their survival.

Prognosis is mainly determined by etiology. Very few complications were reported in relation to anticoagulation treatment.

Further additional investigatory studies are needed in order to identify any better potential treatment.

\section{Funding}

This review did not receive grant from any funding agency in the public, commercial, or non-for-profit sectors.

\section{Conflicts of Interest}

The authors declare no conflicts of interest regarding the publication of this paper.

\section{References}

[1] Stein, P.D., Matta, F. and Yaekoub, A.Y. (2008) Incidence of Vena Cava Thrombosis in the United States. The American Journal of Cardiology, 102, 927-929. https://doi.org/10.1016/j.amjcard.2008.05.046

[2] White, R.H. (2003) The Epidemiology of Venous Thromboembolism. Circulation, 107, I4-I8. https://doi.org/10.1161/01.CIR.0000078468.11849.66

[3] McAree, B.J., O’Donnell, M.E., Fitzmaurice, G.J., Reid, J.A., Spence, R.A. and Lee, B. (2013) Inferior Vena Cava Thrombosis: A Review of Current Practice. Vascular Medicine, 18, 32-43. https://doi.org/10.1177/1358863X12471967

[4] Agnelli, G.L., Verso, M., Ageno, W., Imberti, D., Moia, M., Palareti, G., et al. (2008) The MASTER Registry on Venous Thromboembolism: Description of the Study Cohort. Thrombosis Research, 120, 605-610. https://doi.org/10.1016/j.thromres.2007.06.009

[5] Alkhouli, M., Morad, M., Narins, C., Raza, F. and Bashir, R. (2016) Inferior Vena Cava Thrombosis. JACC: Cardiovascular Interventions, 9, 629-643. https://doi.org/10.1016/i.jcin.2015.12.268

[6] Sitwala, P.S., Ladia, V.M., Brahmbhatt, P.B., Jain, V. and Bajaj, K. (2014) Inferior Vena Cava Anomaly: A Risk for Deep Vein Thrombosis. North American Journal 
of Medical Science, 6, 601-603. https://doi.org/10.4103/1947-2714.145486

[7] Giordano, P., Weber, K., Davis, M. and Carter, E. (2006) Acute Thrombosis of the Inferior Vena Cava. The American Journal of Emergency Medicine, 24, 640-642. https://doi.org/10.1016/j.ajem.2005.12.018

[8] Kraft, C., Hecking, C., Schwonberg, J., Schindewolf, M., Lindhoff-Last, E. and Linnemann, B. (2013) Patients with Inferior Vena Cava Thrombosis Frequently Present with Lower Back Pain and Bilateral Lower-Extremity Deep Vein Thrombosis. Vasa, 42, 275-283. https://doi.org/10.1024/0301-1526/a000288

[9] Shi, W. and Dowell, J. (2017) Etiology and Treatment of Acute Inferior Vena Cava Thrombosis. Thrombosis Research, 149, 9-16.

https://doi.org/10.1016/j.thromres.2016.07.010

[10] Meissner, M.H., Gloviczki, P., Comerota, A.J., Dalsing, M.C., Eklof, B.G., Gillespie, B.L., et al. (2012) Early Thrombus Removal Strategies for Acute Deep Venous Thrombosis: Clinical Practice Guidelines of the Society for Vascular Surgery and the American Venous Forum. Journal of Vascular Surgery, 55, 1449-1462. https://doi.org/10.1016/j.jvs.2011.12.081

[11] Kaufman, L.B., Yeh, B.M., Breiman, R.S., Joe, B.N., Qayyum, A. and Coakley, F.V. (2005) Inferior Vena Cava Filling Defects on CT and MR. American Journal of Roentgenology, 185, 717-726. https://doi.org/10.2214/ajr.185.3.01850717 